\title{
Supervised molecular dynamics for exploring the druggability of the SARS-CoV-2 spike protein
}

\author{
Giuseppe Deganutti ${ }^{1}$ D . Filippo Prischi $^{2} \cdot$ Christopher A. Reynolds $^{1}$
}

Received: 29 July 2020 / Accepted: 15 October 2020 / Published online: 26 October 2020

(c) Springer Nature Switzerland AG 2020

\begin{abstract}
The recent outbreak of the respiratory syndrome-related coronavirus (SARS-CoV-2) is stimulating an unprecedented scientific campaign to alleviate the burden of the coronavirus disease (COVID-19). One line of research has focused on targeting SARS-CoV-2 proteins fundamental for its replication by repurposing drugs approved for other diseases. The first interaction between the virus and the host cell is mediated by the spike protein on the virus surface and the human angiotensin-converting enzyme (ACE2). Small molecules able to bind the receptor-binding domain (RBD) of the spike protein and disrupt the binding to ACE2 would offer an important tool for slowing, or even preventing, the infection. Here, we screened 2421 approved small molecules in silico and validated the docking outcomes through extensive molecular dynamics simulations. Out of six drugs characterized as putative RBD binders, the cephalosporin antibiotic cefsulodin was further assessed for its effect on the binding between the RBD and ACE2, suggesting that it is important to consider the dynamic formation of the heterodimer between RBD and ACE2 when judging any potential candidate.
\end{abstract}

Keywords SARS-CoV-2 · COVID-19 · Drug repurposing $\cdot$ Spike protein $\cdot$ Molecular dynamics $\cdot$ Supervised molecular dynamics

\section{Introduction}

In early 2020, the severe acute respiratory syndrome-related coronavirus (SARS-CoV-2) caused the spread of the coronavirus disease (COVID-19) on an unprecedented global scale. Symptoms comprising smell and taste dysfunction [1], fever, cough, fatigue, headache and dyspnea [2] usually appear after an average incubation period of just over 5 days [3], lasting for 6 to 41 days [4]. The clinical picture is worsened by severe pneumonia with high levels of pro-inflammatory cytokines [2] (a condition also known as "cytokine storm"), which triggers extensive organ damage. SARS-CoV-2 is a

Electronic supplementary material The online version of this article (https://doi.org/10.1007/s10822-020-00356-4) contains supplementary material, which is available to authorized users.

Giuseppe Deganutti

ad5288@coventry.ac.uk

1 Centre for Sport, Exercise and Life Sciences, Coventry University, Coventry CV1 5FB, UK

2 School of Life Sciences, University of Essex, Wivenhoe Park, Colchester CO4 3SQ, UK positive-stranded RNA Betacoronavirus [5] in which about one-third of the genome codes for structural proteins such as envelope proteins, nucleocapsid proteins, membrane proteins and the spike (S) glycoprotein [6].

The S glycoprotein gives the characteristic "crown" shape to the coronaviruses phenotype, by forming prominent structures on the virion envelope, and drives virulence by mediating the first interactions with the host cell [7] and inducing immune responses [8]. The trimeric $\mathrm{S}$ glycoprotein is composed of two subunits, $\mathrm{S} 1$ and $\mathrm{S} 2$, respectively mediating the binding to the host cell and membrane fusion $[9,10]$. Specifically, $\mathrm{S} 1$ comprises the receptor-binding domain (RBD, Fig. 1a) for the angiotensin-converting enzyme 2 (ACE2), and stabilizes the prefusion state of the $\mathrm{S} 2$ subunit, which in turn drives the fusion of the viral particle into the host cell membrane [11]. ACE2 is a type 1 membrane protein with an extracellular peptidase domain (PD) responsible for the maturation of the vasoactive hormone angiotensin [12]. Organs with high expression level of ACE2 are lungs, testis, heart, kidneys, and intestine [13, 14].

An intriguing way to tackle the SARS-CoV-2 infection is to disrupt the entrance of the virus into host cells by blocking the molecular machinery implied in this fundamental 

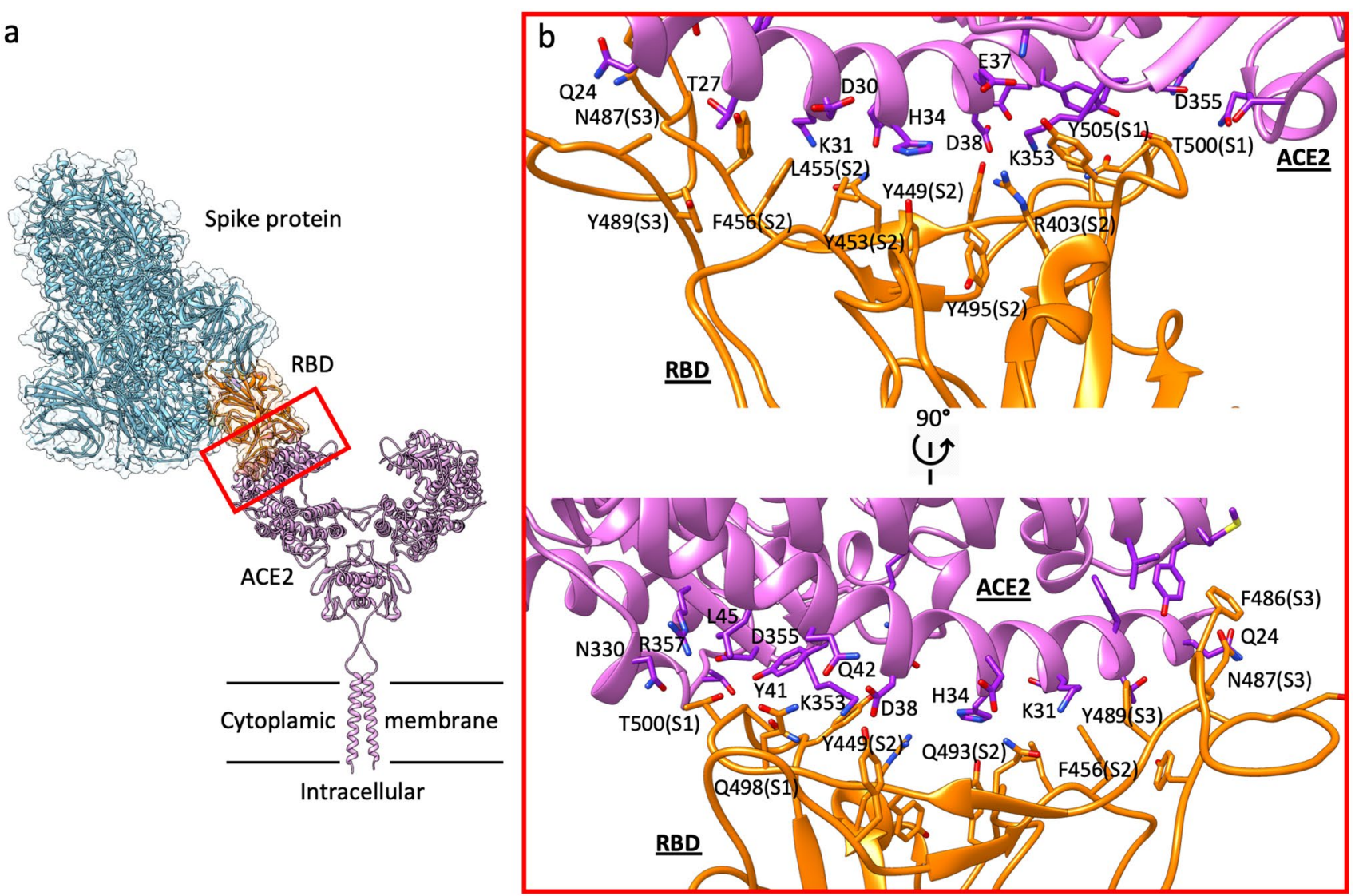

Fig. 1 Supramolecular organization of the spike protein:ACE2 complex and the RBD:ACE2 binding surface. a Schematic representation showing the dimeric ACE2 protruding from the cytoplasmic membrane and acting as receptor for the trimeric SARS-CoV2 spike protein in prefusion open conformation, which binds through one of the three available RBD in up position. $\mathbf{b}$ two side-views of the contact interface between the RBD (orange) and ACE2 (purple). The three RBD sites (S1, S2, and S3) primarily responsible for binding ACE2 $\alpha 1$ helix are indicated within parentheses

costs, due to the low-risk safety profile of already approved drugs [29]. In silico repurposing approaches usually rely on the virtual screening (VS) of small drug databases, followed by a computational validation through molecular dynamics (MD) simulations to evaluate the stability of the predicted complexes in a flexible and fully hydrated simulated environment. Some examples of potential disruptors of the S glycoprotein interaction with ACE2, identified by simple molecular docking of approved drugs include hesperidin [30], paritaprevir [31], cladribine, clofarabine, and fludarabine [32]. Differently, docking combined with MD simulations highlighted denopamine, bometolol, naminterol, rotigaptide, benzquercin [33], simeprevir, lumacaftor [34], tegobuvir, bromocriptine, baicalin [35], KT185, KT203, GSK1838705A, and BMS195614 [36] as potential binders of the RBD.

Assuming some redundancy between the libraries of compounds considered, the lack of consistency from different groups could be considered surprising. However, causes for this discrepancy reside in the RBD coordinates used 
for VS, the different protocols for ligand preparation and docking, the number of MD replicas and the total amount of post-docking MD sampling, the different force fields employed and the free-energy method used for rescoring binding modes. Here, we performed the VS of 2421 FDAapproved drugs against MD-derived coordinates of RBD. Post-docking MD simulations (in triplicate and up to $500 \mathrm{~ns}$ ) of the top-ranked 23 complexes highlighted six promising compounds, which were further evaluated according to the molecular mechanics energies combined with the generalized Born and surface area continuum solvation (MMGBSA) energy. Cefsulodin, which stood out alongside Nilotinib as a potential disruptor of the RBD-ACE2 interface, was then evaluated through supervised molecular dynamics simulations [37-39] (SuMD) aimed to reconstruct the binding event between the RBD and ACE2. Overall, cefsulodin hindered the formation of the heterodimer. However, cefsulodin's affinity for the RBD was affected by metastable interactions with ACE2, indicating the importance of including the heterodimer during the screening of potential candidates.

\section{Methods}

The sequential steps of the computational protocol followed in the present work (Figure S1) are here described.

\section{Classic molecular dynamics simulation of the spike protein RBD}

The coordinates of a monomer of the spike protein (S protein) RBD were retrieved from PDB entry 6M17 [25], which reports the pre-fusion open conformation of the trimeric $\mathrm{S}$ protein (one RBD in up position) in complex with one monomer of ACE2. Only residues C336-F515 from one monomer of the $\mathrm{S}$ protein were considered. After removing the $\mathrm{N}$-acetylglucosamine residues (which are not located in the RBD pocket [22]), hydrogen atoms were added using pdb2pqr [40] and the propka [41] software. The resulting system was prepared for simulations with the Amber14SB [42] force field by creating a $90 \AA \times 92 \AA \times 73 \AA$ TIP3P [43] solvated box. Overall charge neutrality was maintained by adding two $\mathrm{Cl}^{-}$ions.

ACEMD [44] was used for both equilibration and $200 \mathrm{~ns}$ of MD productive simulation. Restraints were applied to protein alpha carbon atoms and gradually released throughout 2 ns of equilibration before starting the MD production. Productive trajectories (Table S4) were computed with an integration time step of $4 \mathrm{fs}$ in the canonical ensemble (NVT). The target temperature was set at $300 \mathrm{~K}$, using a thermostat damping of $0.1 \mathrm{ps}^{-1}$; the M-SHAKE algorithm [45, 46] was employed to constrain the bond lengths involving hydrogen atoms. The cut-off distance for electrostatic interactions was set at $9 \AA$, with a switching function applied beyond 7.5 $⿱$ A. Long range Coulomb interactions were handled using the particle mesh Ewald summation method (PME) [47] by setting the mesh spacing to $1.0 \AA$.

The $200 \mathrm{~ns}$ trajectory was clustered into 10 groups of frames using the VMD plugin Clustering (at $<$ https://githu b.com/luisico/clustering), according to the RMSD of the residues delimiting the RBD pocket (residues Y453, R403, and K417) with respect to the starting conformation. A visual inspection of the trajectory revealed that the breathing of the RBD pocket was primarily due to side chain movements. R403 and K417, in particular, heavily contributed to shaping the pocket because the side chains are longer than those of other neighboring residues. Moreover, position 417 is occupied by valine in the SARS-CoV RBD, indicating a potential element of resistance or selectivity for drug candidates. The frame from the most populated cluster with the highest RMSD value (representative for a "relaxed" and more open conformation of the pocket) was extracted and used for the subsequent steps. This double criterion should allow selection of a structure quite close to the RBD average conformation, taking into account also the possible inducedfit produced by ligand binding, which might favor a more open pocket state.

The RBD from both PDB 6M17 and the MD frame were used as input for the FTMap [48, 49] and DeepSite [50] webservers to evaluate the RBD binding site before and after the MD simulation of RBD (Figure S2). As shown in Figure S2, the binding pocket is larger in the MD structure than in the RBD structure from PDB 6M17.

\section{Virtual Screening of the approved compounds library}

The RBD from the MD frame was used to dock an SDF library of FDA-approved drugs [51] (available at https:// www.selleckchem.com/screening/fda-approved-drug-libra ry.html).

RDKit (https://www.rdkit.org/) was used to remove organometallic compounds and entries with more than 12 rotatable bonds, strip counterions from salt molecules, and generate initial 3D conformations. The resulting database was further processed through Chimera [52] and Open Babel [53] to check the protonation state of the compounds, assign MMFF94 [54] partial charges and generate final pdbqt files for the 2421 structures.

Docking simulations were performed employing AutoDock Vina [55] within a cubic $25 \AA \times 25 \AA \times 25 \AA$ volume centered at the oxygen atom of the Y495 side chain, therefore focusing on the main RBD pocket shown in Figure S2b. Ten conformations for each run were generated. 


\section{Post docking molecular dynamics simulations}

The 23 compounds (Table S1) with the best docking scores were prepared for classic MD with the Amber14SB [42]/ GAFF $[56,57]$ force field by creating a $77 \AA \times 95 \AA \times 71 \AA$ TIP3P [43] solvated box (a padding of $15 \AA$ along the $\mathrm{x}, \mathrm{y}$, and $\mathrm{z}$ dimensions was considered). Overall charge neutrality was maintained by adding $\mathrm{Cl}^{-}$or $\mathrm{Na}^{+}$counterions. ACEMD [44] was used for both equilibration and three replicas of duration $100 \mathrm{~ns}$ each. Restraints were applied to protein alpha carbon atoms and gradually released throughout $2 \mathrm{~ns}$ of equilibration before starting the MD production replicas.

After $100 \mathrm{~ns}$, the RMSD of the compounds with respect to the docking pose was calculated with VMD [58] after aligning the RBD alpha carbon atoms to the initial conformation. The MD replicas in which the final ligand RMSD was lower than $7 \AA$ were extended to $500 \mathrm{~ns}$, for a total of 13 replicas involving 9 compounds (Table S1). The RMSD of the drugs was then computed again, highlighting the compounds still bound to the RBD pocket (Table S1): cefsulodin, cromoglycate, nafamostat, nilotinib, penfluridol, and radotinib.

For these six compounds, the binding energies were evaluated by computing the molecular mechanics energies combined with the generalized Born and surface area continuum solvation were computed with the MMPBSA.py [59] script, from the AmberTools17 suite (The Amber Molecular Dynamics Package, at https://ambermd.org/) employing the default settings and considering the last $50 \mathrm{~ns}$ of simulations out of $500 \mathrm{~ns}$ ( 1 frame every 100 ps of the simulation).

\section{Classic MD simulations of ACE2:RBD complex}

The coordinates of the spike protein RBD (residues C336-F515 from one monomer of the S protein) and human ACE2 (residues I21-N599) were retrieved from one monomer present in the PDB entry 6M17 [25]. After removing the $\mathrm{N}$-acetylglucosamine residues (which are not located at the interface between the proteins) and the zinc ion, hydrogen atoms were added using pdb2pqr [40] and the propka [41] software. The resulting system was prepared for simulations with the Amber14SB [42] force field by creating a $124 \AA \times 117 \AA \times 160 \AA$ TIP3P [43] solvated box (a padding of $15 \AA$ along the $\mathrm{x}, \mathrm{y}$, and $\mathrm{z}$ dimensions was considered). Overall charge neutrality was maintained by adding $21 \mathrm{Na}^{+}$ ions.

ACEMD [44] was used for both equilibration and $200 \mathrm{~ns}$ of MD productive simulation. Restraints were applied to protein alpha carbon atoms and gradually released throughout 2 ns of equilibration before starting the MD production.

MMGBSA binding energies were computed as described in Sect. "Post Docking molecular dynamics simulations" considering 1000 frames ( 1 frame every 200 ps of the simulation).

\section{Supervised MD simulations of the ACE2:RBD complex}

The supervised molecular dynamics (SuMD) is an adaptive sampling method for speeding up the simulation of binding [37, 39] and unbinding processes [38, 60]. Sampling is gained without the introduction of any energetic bias, by applying a tabu-like algorithm to monitor the distance between the centers of mass (or the geometrical centers) of the ligand and the predicted binding site or the receptor. However, the supervision of a second metric of the system can be considered [60]. A series of short unbiased MD simulations are performed, and after each simulation, the distances (collected at regular time intervals) are fitted to a linear function. If the resulting slope is negative (for binding) or positive (for unbinding) the next simulation is started from the last set of atom velocities and coordinates, otherwise, the simulation is discarded and a new one is started from the last productive coordinates by randomly assigning the atomic velocities.

The RBD (residues C336-F515 from one monomer of the S protein) and ACE2 (residues I21-N599) from one monomer present in the PDB entry 6M17 [25] were placed about $25 \AA$ away from each other and prepared for simulations as reported in Sect. "Classic molecular dynamics simulation of the spike protein RBD”. Restraints were applied to protein alpha carbon atoms and gradually released throughout $2 \mathrm{~ns}$ of equilibration before starting the MD production simulation. Four SuMD replicas were collected by monitoring the distance between the centroid of RBD residue Q493 and the centroid of ACE2 residues K31, E35, throughout successive 2 ns-long windows. In the PDB structure 6M17, the interactions between Q493 (RBD) and K31, E35 (ACE2) are in a central position at the interface between proteins. The supervision was iterated until the distance was lower than $7 \AA$, then 200 ns of classic (unsupervised) MD was performed.

MMGBSA binding energies were computed as described in Sect. Post Docking molecular dynamics simulations considering 1 frame every 100 ps of the simulation.

\section{SuMD simulations of the RBD:cefsulodin:ACE2 ternary complex}

The RBD:cefsulodin complex resulting from the post docking MD (500 ns, Sect. "Classic MD simulations of ACE2:RBD complex") was placed about $25 \AA$ away from ACE2 (residues I21-N599). The resulting system was prepared for simulations as reported in Sect. Classic MD simulations of ACE2:RBD complex. In analogy with SuMD simulations in the absence of cefsulodin bound to RBD 
(Sect. SuMD simulations of the RBD:cefsulodin:ACE2 ternary complex), four SuMD replicas were collected by monitoring the distance between the centroid of RBD residue Q493 and the centroid of ACE2 residues K31, E35, throughout successive 2 ns-long windows. The supervision was iterated until the distance was lower than $7 \AA$, then 200 ns of classic (unsupervised) MD was performed.

Molecular mechanics energies combined with the generalized Born and surface area continuum solvation were computed with the MMPBSA.py [59] script, from the AmberTools17 suite (The Amber Molecular Dynamics Package, at https://ambermd.org/) employing the default settings and considering 1 frame every 100 ps of simulation.

\section{Results and discussion}

\section{Virtual screening and post-docking molecular dynamics simulations suggest putative SARS-CoV-2 RBD binders amongst FDA-approved drugs}

Virtual screening (VS) of approved compounds represents the first step of drug repurposing strategies in the pursuit of candidates able to target SARS-CoV-2. Amongst the viral proteins that could be therapeutically exploted [30, 61], targeting the $\mathrm{S}$ glycoprotein could represent a promising way to treat and, most importantly, prevent COVID-19. Indeed, the ACE2:RBD binding interface (Fig. 1), partially overlaps a pocket (Figure S2) that can be targeted with small molecules to disrupt the early stage of SARS-CoV-2 infection.

The use of MD structures, instead of cryo-EM or crystal structures, can enhance the docking predictivity [62] thanks to the relaxation of the system from the solid phase to the fully hydrated one and the inclusion of full molecular flexibility. Extracting the RBD coordinates from the cryo-EM structure of the RBD-ACE2 complex (PDB 6M17) and processing the RBD through MD simulations allowed the RBD pocket to expand (Figure S1a, b) and to expose residues located within site 2 (Figure S1c, d). On this optimized structure, we docked 2421 approved drugs. The 23 topranked compounds from the VS (Table S1) were subjected to three MD replicas to validate the stability of the predicted complexes. Performing MD simulations from docking poses represents a valuable approach for evaluating the stability of docking-predicted complexes in a fully hydrated and flexible environment, therefore overcoming well-known limits of molecular docking [63].

Notably, none of these 23 drugs remained bound to RBD in all the three MD replicas (Table S1). The best-ranked compound according to Autodock, dihydroergotamine, completely dissociated during one replica, and moved away in the other two replicas, transiently interacting with other regions of the RBD (final RMSDs with respect to the docked pose were $25.6 \AA$ and $14.5 \AA$ respectively). The secondranked compound, nilotinib, displayed good stability with slight conformational rearrangement throughout the three MD simulations (final RMSDs with respect to the docked pose $3.5 \AA$, $3.8 \AA$ and $5.4 \AA$ respectively). Cefsulodin and cromoglycate remained stably bound during two MD replicas out of three, while fluralaner, nafamostat, naringin, penfluridol, radotinib, and regorafenib were stable during one replica (Table S1).

The 13 simulations characterized by good stability were extended to $500 \mathrm{~ns}$ for further evaluating the stability of the complexes. Fluralaner, naringin, and regorafenib dissociated from the RBD (final RMSD with respect to the docked pose 14.4 ̊, 11.8 $\AA$ and $12.4 \AA$, respectively). Six compounds, on the other hand, remained bound: cefsulodin, cromoglycate, nafamostat, nilotinib, penfluridol, and radotinib (Table S1, Figure S3a). MMGBSA binding energies (Table S1, Fig. 3Sb) indicated nilotinib and cefsulodin as the most stable ligands $(-53.2 \pm 4.1 \mathrm{kcal} / \mathrm{mol}$ and $-41.3 \pm 6.7 \mathrm{kcal} / \mathrm{mol}$, respectively), while the other compounds displayed a similar MMGBSA binding energy (e.g. values close to $-30 \mathrm{kcal} /$ mol). A comparison of the last frames from the six $500 \mathrm{~ns}$ MD simulations reveals similarities and differences in the conformations of the bound ligands and the RBD (Fig. 2a). Direct interactions with cromoglycate and nafamostat, for example, folded RBD site 3 towards the putative binding pocket occupied by the ligand. As a general view, the six compounds occupied a pocket region partially overlapping RBD site 1 and site 2 , and formed four main groups of interactions with the protein (Fig. 2b). Within RBD site 1, the ligands formed hydrophobic $\pi-\pi$ interactions with the side chain of Y505 and hydrogen bonds with the hydrophilic region in the proximity of backbone and the side chain of N501. Site 2, instead, stabilized the ligands through a mix of hydrophobic interactions deep in the pocket and hydrogen bonds with residues on the surface of the binding site (which is comprised of R403, K417, Y453, and G496).

\section{Cefsulodin, cromoglycate, nafamostat, nilotinib, penfluridol and radotinib proposed binding modes}

Cefsulodin (Fig. 2c) is a third-generation $\beta$-lactam cephalosporin antibiotic with a spectrum of activity restricted to Pseudomonas aeruginosa and Staphylococcus aureus [64]. Interestingly, during the $500 \mathrm{~ns}$ MD simulation, cefsulodin rapidly dissociated from the starting docked pose before rebinding the RBD pocket after $100 \mathrm{~ns}$ with a different stable conformation (MMGBSA binding energy $-41.3 \pm 6.7 \mathrm{kcal} /$ mol, Table S1), Figure S3a, Video S1). In this binding mode, the drug formed hydrogen bonds with G502, G496, R403, and $\mathrm{K} 417$, and hydrophobic contacts with Y505, K417, I418, Y453, and Y495 side chains (Fig. 2c, Video S1). 

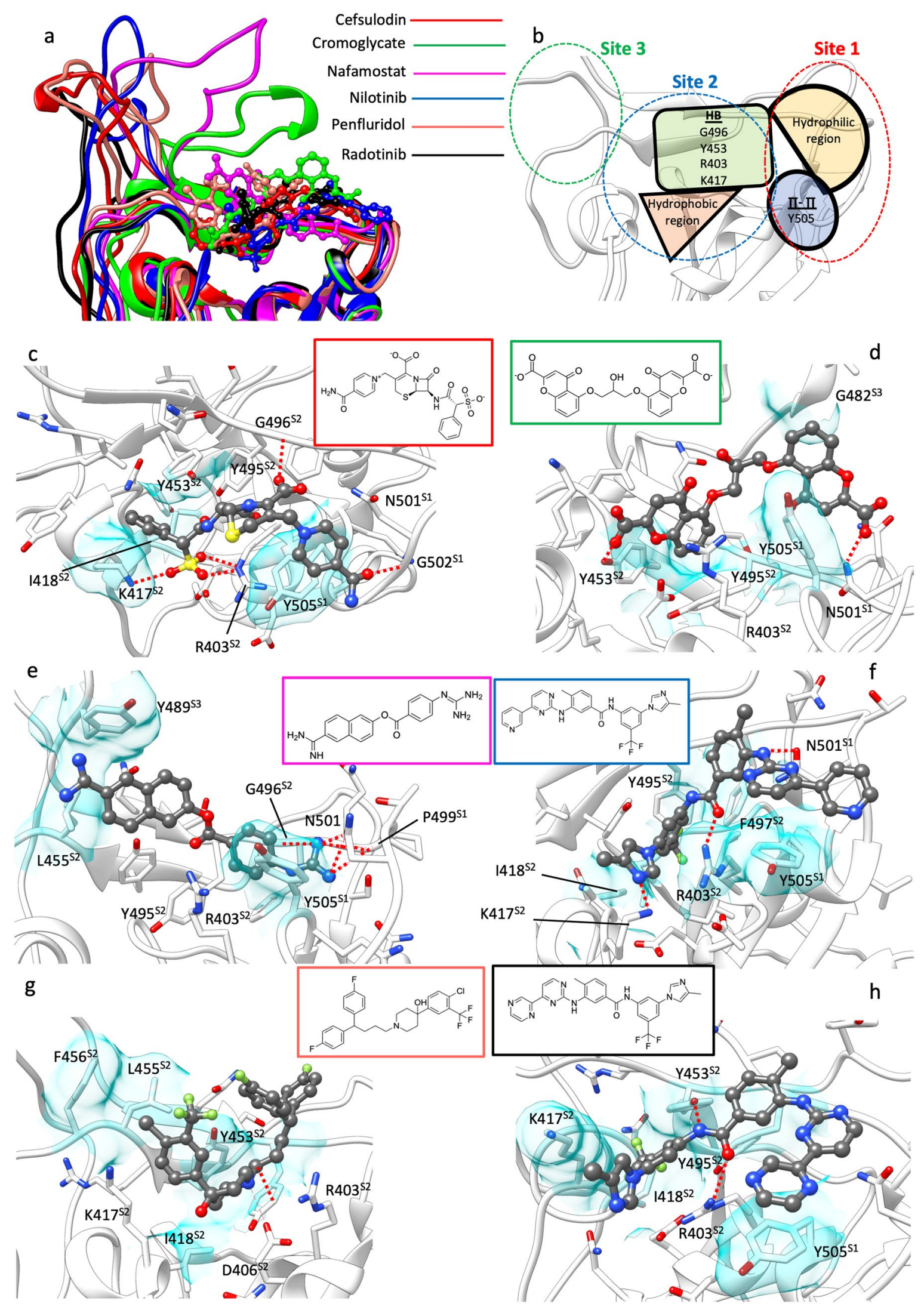
4Fig. 2 Binding conformation of cefsulodin, cromoglycate, nafamostad, nilotinib, penfluridol, and radotinib after 500 ns of post-docking MD simulations. a comparison between the last MD frames highlighting the different RBD orientation; cromoglycate and nafamostat strongly affected the RBD site 3 . b schematic representation of the interaction features characterizing the RBD pocket as determined by the binding modes of the six proposed ligands; the three sites responsible for binding ACE2 are indicated with dashed lines (site 1 in red, site 2 in blue, and site 3 in green respectively). c-h binding modes of the ligands after $500 \mathrm{~ns}$ of post-docking classic MD. Hydrogen bonds are shown as dashed red lines, while hydrophobic contacts are depicted as transparent cyan surfaces. The 2D structures of the compounds are reported for clarity. c) cefsulodin; $\mathbf{d}$ cromoglycate: e nafamostat; f nilotinib; $\mathbf{g}$ penfluridol; $\mathbf{h}$ radotinib. The three RBD sites responsible for binding ACE2 are indicated with superscripts on the RBD residue names ( $\mathrm{S} 1, \mathrm{~S} 2$, and $\mathrm{S} 3$ )

Interestingly, the other cephalosporins that were docked all obtained modest scores. The analog closer to cefsulodin in term of docking results was cefonicid (docking score $=-7.0 \mathrm{kcal} / \mathrm{mol}$, Figure S4). A direct comparison between cefsulodin, cefonicid and cefazolin (a further $\beta$-lactam cephalosporin, modest docking score of $-6.2 \mathrm{kcal} /$ mol, Figure S4) shows how different structures affected docking results: cefsulodin and cefonicid, indeed, bear a terminal phenyl ring which appeared to drive a common docking pose, while cefazolin's tetrazole moiety produced a divergent flipped predicted conformation (Figure S4).

Cromoglycate (Fig. 2d) is an anti-inflammatory drug indicated for the prevention of acute episodes in patients with mild to moderate asthma. During the 500 ns post-docking MD simulation cromoglycate experienced remarkable fluctuations within the binding site (Figure S3, Video S2) before forming interactions with residues that are part of RBD site 3 (i.e. G482, Fig. 2d, Fig. 2a, Video S2). The drug formed two persistent hydrogen bonds between the carboxylates and N501, Y453 side chains (Fig. 2d). Hydrophobic interactions involved Y505, Y495, and Y453 (Fig. 2d).

Nafamostat (Fig. 2e) is a serine protease inhibitor currently in clinical trials for SARS-CoV2 infection in the light of its proposed ability to inhibit the transmembrane protease serine 2 (TMPRSS2)-dependent host cell entry [65, 66]. During our simulation (Video S3) nafamostat remained bound to the RBD pocket thanks to hydrogen bonds between the guanidinium group and N501, G502 and G496 (Fig. 2e), as well as hydrophobic contacts with Y505 (MMGBSA binding energy $=-29.8 \pm 3.9$ ). Transient interactions were formed between the molecule and the RBD site 3, in particular the Y489 side chain (Fig. 2e, Video S3).

Nilotinib (Fig. 2f) is a tyrosine kinases inhibitor with antineoplastic activity. In has been proposed to act in two different stages of the coronavirus infection cycle: in the early phases of infection, it has been proposed to inhibit the virion fusion with the endosome ${ }^{1}$, while at a later stage it has been proposed to inhibit the viral replication [67] via
ABL-mediated cytoskeletal rearrangement [68, 69]. During the 500 ns MD simulation (Video S4), nilotinib, which remained stably bound to the RBD pocket (MMGBSA binding energy $=-53.2 \pm 4.1 \mathrm{kcal} / \mathrm{mol}$ ), positioned the trifluoromethyl group deep in the binding pocket and formed hydrophobic interactions with I418, Y495, F497, Y505, and the alkyl chain of R403 (Fig. 2f, Video S4). Hydrogen bonds were established between the drug and the N501 and R403 side chains (Fig. 2f, Video S4).

Penfluridol (Fig. 2g) is a long-acting antipsychotic agent [70]. During the 500 ns MD simulation (Video S5) the drug was anchored to the RBD through interactions between the piperidin-4-ol group and residues D406, Y453 (Fig. 2g, Video S5), while the flexible bis (4-fluorophenyl)butyl and 4-chloro-3-(trifluoromethyl)phenyl moieties explored several conformations. Notably, penfluridol shares the phenylpiperidin-4-ol scaffold with the SARS-CoV inhibitor K22 [71], which may indicate a common molecular mechanism.

Radotinib (Fig. 2h) is a second-generation BCR-ABL tyrosine kinase inhibitor with therapeutic indication for patients resistant or intolerant to imatinib [72]. Despite the high structural similarity with nilotinib (from which it differs only by the pyridine terminal ring, Fig. $2 f$, h) and the good interaction network within the RBD pocket (hydrogen bonds with R403 and Y453 side chains, hydrophobic interactions with Y505, I418, and Y495, Fig. 2h), radotinib displayed lower stability (MMGBSA binding energy $=-28.6 \pm 3.8$ ) and higher conformational fluctuation (Video S6) than nilotinib. The reason for this could reside in the different conformations predicted by docking (Figure S5). Indeed, the nilotinib top-ranked pose oriented the trifluoromethyl group toward the inner side of the pocket, while radotinib's best pose was predicted to be almost planar on the pocket surface. This binding mode of radotinib did not evolve towards more stable states during the MD simulation (e.g. it did not reorient the trifluoromethyl group), resulting in sub-optimal interactions with the RBD. The different outcomes from almost identical ligands highlights the importance of the docking pose choice. The common practice to pick the top-ranked pose can lead to overlooking other suitable conformations that are penalized by lower docking scores computed without considering the explicit solvent contribution to the binding. However, the MD post-processing of docking poses can be time-consuming, and therefore applicable only to a small set of compounds $[63,73]$ on the basis of the ranking provided by docking scoring functions.

\section{Simulating the RBD:ACE2 binding mechanism to retrieve insights into the effect of potential disruptors}

During post-docking MD simulations, putative binders of the RBD interacted with protein residues that are responsible 
RMSD - RBD

RMSD - RBD binding interface

RBD:ACE2 GBSA Energy

Cefsulodin GBSA Energy

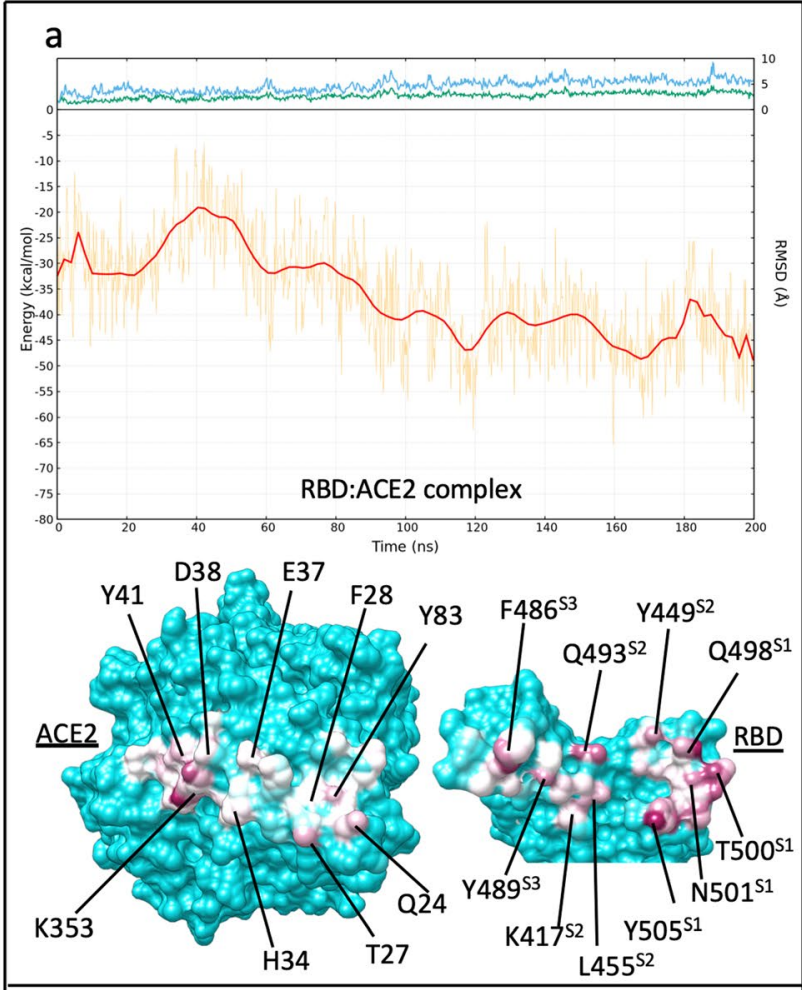

c
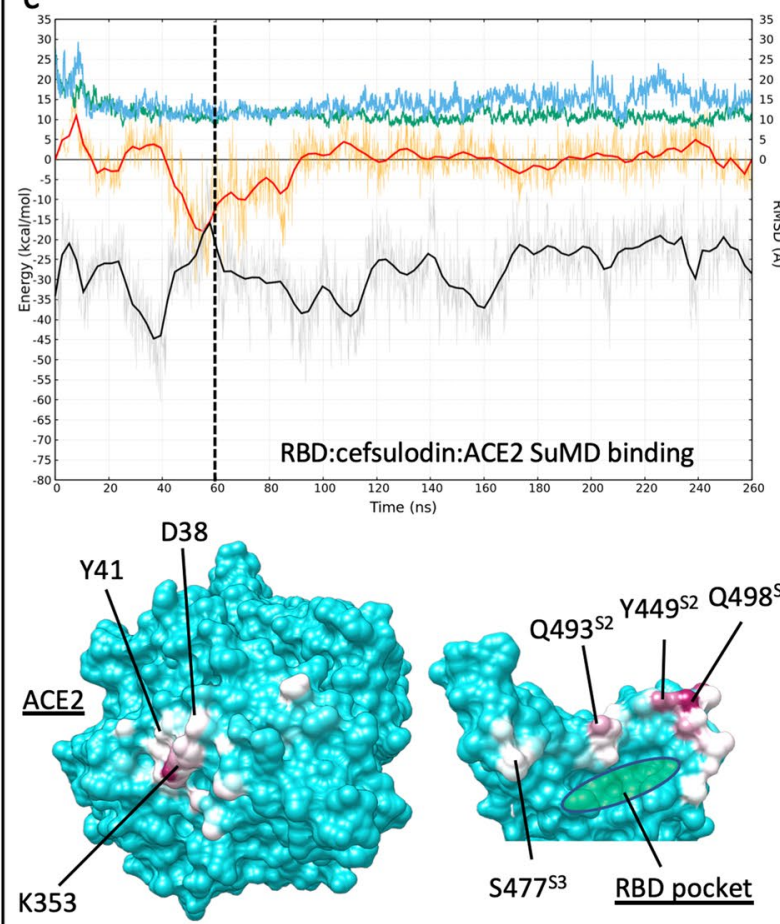

b
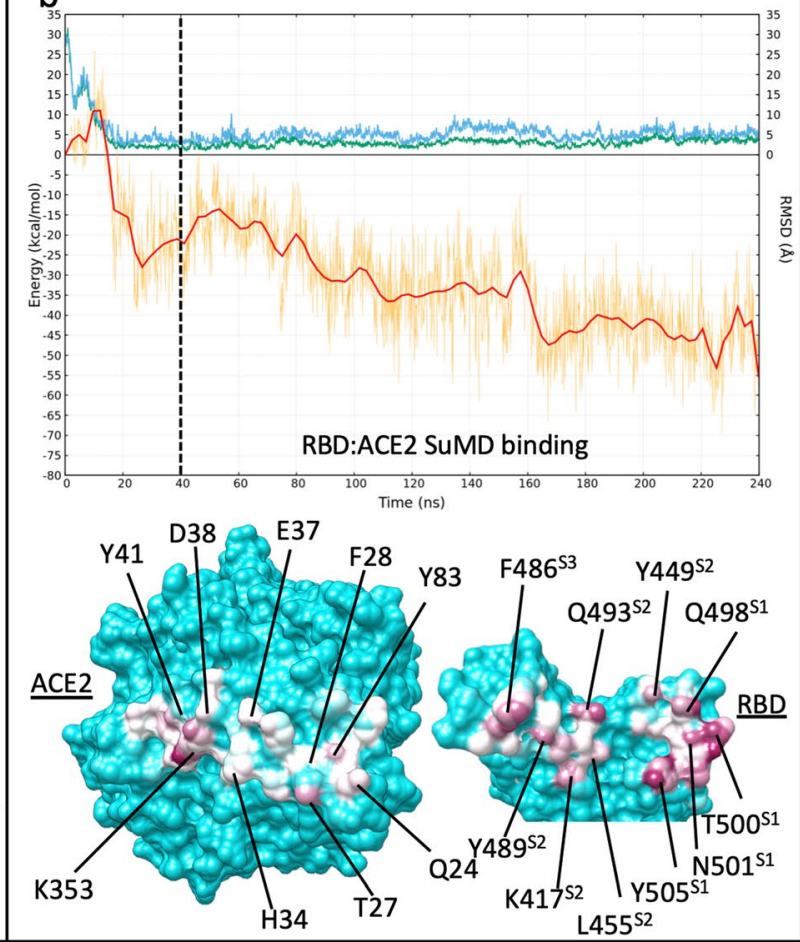

d
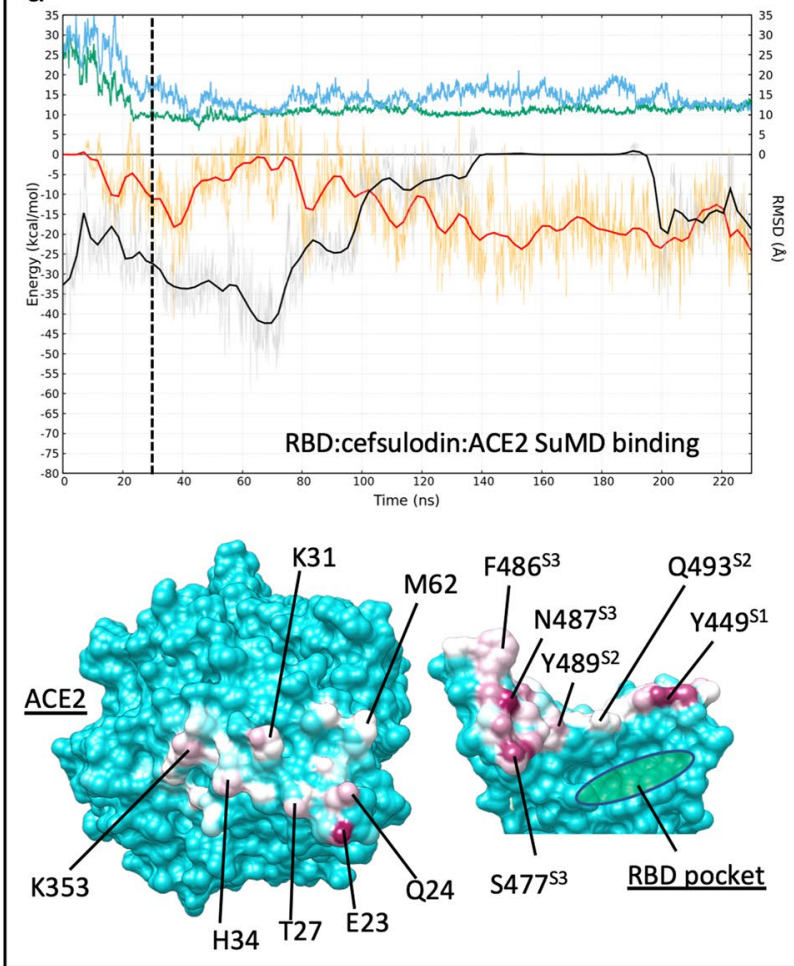
4 Fig. 3 Influence of RBD-bound cefsulodin on the intermolecular recognition between RBD and ACE2 during binding. Top panels plots show the RMSD with respect to PDB 6M17 of the whole RBD (cyan line) and the RBD residues located at the binding interface with ACE2 (green line), respectively; the red and black lines indicate the MMGBSA binding energy of RBD:ACE2 complex and cefsulodin, respectively. Bottom panels: RBD:ACE2 intermolecular contacts potted on the surface of the proteins; the RBD pocket, which was occupied by cefsulodin at the beginning of the simulations, is indicated in green in (c) and (d). a classic MD simulation of the RBD:ACE2 complex from PDB 6M17; b RBD:ACE SuMD binding; the dimer reached the $6 \mathrm{M} 17$ bound conformation (RMSD $\approx 2 \AA$ ) in less than 20 ns. c)) RBD:cefsulodin:ACE ternary complex SuMD binding replica 1; despite reaching the ACE2 surface, RBD was not stabilized due to the co-presence of cefsulodin on protein interfaces; $\mathbf{d}$ RBD:cefsulodin:ACE2 ternary complex SuMD binding replica 2; cefsulodin was displaced by ACE2 after $140 \mathrm{~ns}$ of simulation, However, the RBD did not reach the experimental conformation within 230 ns (RMSD $\approx 10-15 \AA$ ).). Vertical dashed lines indicate the start of $200 \mathrm{~ns}$ of classic MD after SuMD; the three RBD sites responsible for binding ACE2 are indicated with superscripts on the RBD residue names (S1, S2, and S3)

for direct contacts with ACE2 (Figs. 1,2). Cefsulodin and nilotinib, for example, made stable contacts with residues located on both RBD site 1 (Y505, N501, G502, and F497) and site 2 (L455, Y495 and G496) that contributes to the stabilization of the complex with ACE2 (Table S2). This indicates the potential ability of these drugs to disrupt key interactions of the SARS-CoV-2 spike protein. To further test this hypothesis, we simulated the binding between the RBD and ACE2 in the absence of any small molecule or in presence of cefsulodin bound to RBD (conformation sampled after $500 \mathrm{~ns}$ of post-docking MD, Fig. 2c). We focused on cefsulodin rather than nilotinib in the light of the spontaneous re-binding event observed during the simulation (Video S1, Figure S3), the numerous hydrogen bonds formed with the RBD (Fig. 2c), and the low toxicity [74].

The first batch of SuMD simulations sought to reproduce a putative binding path of the RBD:ACE2 hetero dimer (PDB 6M17). In one out of four replicas, the RBD was able to bind in close agreement with the experimental conformation reported in the PDB structure 6M17 (Video S7, Fig. 3a, b). A further SuMD replica reproduced the complex with good agreement to the cryo-EM structure (Figure S6a), while two other simulations were not productive after $230 \mathrm{~ns}$ (Figure S6b, c). SuMD reproduced the stability and intermolecular contacts observed between the RBD and ACE2 during MD of the PDB structure 6M17 (Fig. 3a, b), highlighting RBD site 1 residues T500, Y505, N501, and Q498 as the most involved in interactions alongside Q493, L455, K417 (site 2) and F486 (site 3) [34, 75, 76].

These data represented a robust reference for evaluating the potential effect of a ligand on the formation of the RBD:ACE complex. We then performed four SuMD replicas to study the binding between the RBD in complex with cefsulodin and ACE2 (Video S8, Video S9, Fig. 3c, d, Figure
S7). During two simulations, the RMSD of the RBD with respect to the experimental conformation increased, indicating non-productivity of the simulated binding event due to stochastic reasons (e.g. the supervision on the distance between the RBD residue Q493 and the ACE2 residues K31 and E35 failed to move the proteins closer), rather than the presence of cefsulodin (Figure S7). The other two replicas, instead, were characterized by RMSD values decreasing to a plateau of about 10-15 $\AA$ (Fig. 3c, d), consistent with a possible productive contact between the proteins. In one of the two productive SuMD simulations (Video S8, Fig. 3c), despite the formation of a ternary complex involving the RBD, cefsulodin and ACE2, the MMGBSA binding energy between the RBD and ACE2 oscillated around zero, indicating the formation of a sub-optimal binding interface between the two proteins (Fig. 3c). Following a slight conformational rearrangement, cefsulodin remained bound to the interface between proteins for the whole simulation (MMGBSA binding energy in the $-20--40 \mathrm{kcal} / \mathrm{mol}$ range, Fig. 3c, cf. MMGBSA binding energy in the range of $-32--50 \mathrm{kcal} /$ mol in Figure S3). The RBD formed limited contacts with ACE2 (residues D38, Y41, and K353, Fig. 3c) through Y449 and Q498 (site 1), and Q494 (site 2) side chains. A divergent scenario was sampled during the other productive SuMD replica (Video S9, Fig. 3d). During this simulation, cefsulodin was displaced by ACE2 after $140 \mathrm{~ns}$, allowing the formation of a metastable binary complex between RBD and ACE2 (RBD-ACE2 MMGBSA binding energy $\approx-20 \mathrm{kcal} /$ mol, against $\approx-45 \mathrm{kcal} / \mathrm{mol}$ as computed for the experimental complex, Fig. 3a,b); RBD residues F486, N487, S477 (site 3), Y489 Q493 (site 2), and T449 (site 1) were highly involved. Despite the displacement of cefsulodin, the RBD did not reach the experimental conformation over the simulated $230 \mathrm{~ns}$ (RMSD $\approx 10-15 \AA$ ). However, over a longer timescale, it is plausible that the RMD eventually would rearrange to the fully bound conformation.

Taken together, these results suggest that a compound selected through VS and post-docking MD simulations may be less effective when the dynamic of protein-protein formation is taken into account. SuMD (and other adaptive or enhanced MD sampling methods) may represent a further step in silico to the identification of protein-protein interaction (PPI) disruptors that may ultimately make the design process more effective.

\section{Conclusion}

We performed the VS and extensive MD and SuMD simulations of FDA-approved drugs. According to our computational protocol, six compounds (cefsulodin, cromoglycate, nafamostat, nilotinib, penfluridol, and radotinib) are predicted to bind the SARS-CoV-2 S glycoprotein RBD. Cefsulodin (a 
cephalosporin antibiotic) and nilotinib (a kinases inhibitor) were the most efficient binders in silico.

We further evaluated cefsulodin as a potential disruptor of the RBD:ACE2 complex by simulating the binding process in the absence and presence of the drug bound to the RBD. While cefsulodin hindered the formation of the complex in the simulated conditions, it also appears that ACE2 altered the affinity of cefsulodin for the RBD. According to SuMD, the presence of cefsulodin in the RBD pocket during the approach to ACE2 could modify the overall binding path between the two proteins, hampering the formation of the experimental arrangement observed in the cryo-EM structure. This is promising for the success of drug repurposing strategies targeting the early step of SARS-CoV-2 infection. However, our simulations suggested also the alternative scenario in which the dynamic protein-protein interface formed during the binding between RBD and ACE2 creates a supramolecular environment that modifies the affinity of the putative disruptor for the RBD pocket, with the final effect of partially or completely displacing it. This double outline raises the question whether disruptors should be in silico designed considering only complementarity and affinity for the RBD pocket or designed to stabilize unproductive metastable ternary complexes (e.g. involving the RBD, ACE2 and the ligand). Future work will aim to compare several other potential RBD binders (i.e. nilotinib) to deliver insights about what set of interactions with the RBD are more prone to stabilize the ligand during the ACE2 recognition.

If repurposing strategies fail to deliver an actual disrupter of the protein, it would be necessary to further investigate the binding process between the spike protein and ACE2, characterizing intermediate complexes that could be drugged to prevent the formation of the one which is productive for the cell invasion. These results extend the computational toolkit for the rational discovery of PPIs disruptors to adaptive or enhanced sampling methods, like SuMD, able to simulate the nonequilibrium formation of homo- and hetero dimers.

Funding CAR and GD received funding from Leverhulme Trust Grant No. RPG-2017-255.

Data availability Topology files and MD trajectories are available at: https://zenodo.org/record/3997178\#.X4Aw9ZNKjt2

\section{Compliance with ethical standards}

Conflict of interest The authors declare no conflict of interests.

\section{References}

1. Xydakis MS, Dehgani-Mobaraki P, Holbrook EH, Geisthoff UW, Bauer C, Hautefort C, Herman P, Manley GT, Lyon DM, Hopkins C (2020) Smell and taste dysfunction in patients with COVID-19.
Lancet Infect Dis 20:1015-1016. https://doi.org/10.1016/S1473 -3099(20)30293-0

2. Huang C, Wang Y, Li X, Ren L, Zhao J, Hu Y, Zhang L, Fan G, Xu J, Gu X, Cheng Z, Yu T, Xia J, Wei Y, Wu W, Xie X, Yin W, Li H, Liu M, Xiao Y, Gao H, Guo L, Xie J, Wang G, Jiang R, Gao Z, Jin Q, Wang J, Cao B (2020) Clinical features of patients infected with 2019 novel coronavirus in Wuhan, China. Lancet 395:497-506. https://doi.org/10.1016/S0140-6736(20)30183-5

3. Rothan HA, Byrareddy SN (2020) The epidemiology and pathogenesis of coronavirus disease (COVID-19) outbreak. J Autoimmun 109:102433. https://doi.org/10.1016/j.jaut.2020.102433

4. Wang W, Tang J, Wei F (2020) Updated understanding of the outbreak of 2019 novel coronavirus (2019-nCoV) in Wuhan, China. J Med Virol 92:441-447. https://doi.org/10.1002/jmv.25689

5. $\mathrm{Li} \mathrm{F}$ (2016) Structure, function, and evolution of coronavirus spike proteins. Annu Rev Virol 3:237-261. https://doi.org/10.1146/ annurev-virology-110615-042301

6. Fehr AR, Perlman S (2015) Coronaviruses: an overview of their replication and pathogenesis. Methods Mol Biol 1282:1-23. https ://doi.org/10.1007/978-1-4939-2438-7_1

7. Collins AR, Knobler RL, Powell H, Buchmeier MJ (1982) Monoclonal antibodies to murine hepatitis virus-4 (strain JHM) define the viral glycoprotein responsible for attachment and cell-cell fusion. Virology 119:358-371. https://doi.org/10.1016/00426822(82)90095-2

8. Jiang F, Deng L, Zhang L, Cai Y, Cheung CW, Xia Z (2020) Review of the clinical characteristics of Coronavirus disease 2019 (COVID-19). J Gen Intern Med 35:1545-1549. https://doi. org/10.1007/s11606-020-05762-w

9. Abraham S, Kienzle TE, Lapps W, Brian DA (1990) Deduced sequence of the bovine coronavirus spike protein and identification of the internal proteolytic cleavage site. Virology 176:296301. https://doi.org/10.1016/0042-6822(90)90257-r

10. Hoffmann M, Kleine-Weber H, Pöhlmann S (2020) A multibasic cleavage site in the spike protein of SARS-CoV-2 is essential for infection of human lung cells. Mol Cell 78:779-784.e5. https:// doi.org/10.1016/j.molcel.2020.04.022

11. Walls AC, Park Y-J, Tortorici MA, Wall A, McGuire AT, Veesler D (2020) Structure, function, and antigenicity of the SARS-CoV-2 spike glycoprotein. Cell 181:281-292.e6. https://doi.org/10.1016/j. cell.2020.02.058

12. Tikellis C, Thomas MC (2012) Angiotensin-converting enzyme 2 (ACE2) is a key modulator of the renin angiotensin system in health and disease. Int J Pept 2012:256294. https://doi. org/10.1155/2012/256294

13. Donoghue M, Hsieh F, Baronas E, Godbout K, Gosselin M, Stagliano N, Donovan M, Woolf B, Robison K, Jeyaseelan R, Breitbart RE, Acton S (2000) A novel angiotensin-converting enzyme-related carboxypeptidase (ACE2) converts angiotensin I to angiotensin 1-9. Circ Res 87:E1-9. https://doi.org/10.1161/01. res.87.5.e1

14. Li M-Y, Li L, Zhang Y, Wang X-S (2020) Expression of the SARS-CoV-2 cell receptor gene ACE2 in a wide variety of human tissues. Infect Dis Poverty 9:45. https://doi.org/10.1186/s4024 9-020-00662-X

15. Joshi S, Joshi M, Degani MS (2020) Tackling SARS-CoV-2: proposed targets and repurposed drugs. Future Med Chem. https:// doi.org/10.4155/fmc-2020-0147

16. Tiwari V, Beer JC, Sankaranarayanan NV, Swanson-Mungerson M, Desai UR (2020) Discovering small-molecule therapeutics against SARS-CoV-2. Drug Discov Today. https://doi. org/10.1016/j.drudis.2020.06.017

17. Whisenant J, Burgess K (2020) Blocking Coronavirus 19 infection via the SARS-CoV-2 spike protein: initial steps. ACS Med Chem Lett 11:1076-1078. https://doi.org/10.1021/acsmedchem lett.0c00233 
18. Xiu S, Dick A, Ju H, Mirzaie S, Abdi F, Cocklin S, Zhan P, Liu $X$ (2020) Inhibitors of SARS-CoV-2 entry: current and future opportunities. J Med Chem. https://doi.org/10.1021/acs.jmedc hem.0c00502

19. Chi X, Yan R, Zhang J, Zhang G, Zhang Y, Hao M, Zhang Z, Fan P, Dong Y, Yang Y, Chen Z, Guo Y, Zhang J, Li Y, Song X, Chen Y, Xia L, Fu L, Hou L, Xu J, Yu C, Li J, Zhou Q, Chen W (2020) A neutralizing human antibody binds to the $\mathrm{N}$-terminal domain of the Spike protein of SARS-CoV-2. Science 369:650-655. https://doi.org/10.1126/science.abc6952

20. Pinto D, Park Y-J, Beltramello M, Walls AC, Tortorici MA, Bianchi S, Jaconi S, Culap K, Zatta F, De Marco A, Peter A, Guarino B, Spreafico R, Cameroni E, Case JB, Chen RE, Havenar-Daughton C, Snell G, Telenti A, Virgin HW, Lanzavecchia A, Diamond MS, Fink K, Veesler D, Corti D (2020) Cross-neutralization of SARS-CoV-2 by a human monoclonal SARS-CoV antibody. Nature 583:290-295. https://doi.org/10.1038/s4158 6-020-2349-y

21. Wang C, Li W, Drabek D, Okba NMA, van Haperen R, Osterhaus ADME, van Kuppeveld FJM, Haagmans BL, Grosveld F, Bosch B-J (2020) A human monoclonal antibody blocking SARS-CoV-2 infection. Nat Commun 11:2251. https://doi.org/10.1038/s4146 7-020-16256-y

22. Chen Y, Guo Y, Pan Y, Zhao ZJ (2020) Structure analysis of the receptor binding of 2019-nCoV. Biochem Biophys Res Commun. https://doi.org/10.1016/j.bbrc.2020.02.071

23. Ho T-Y, Wu S-L, Chen J-C, Wei Y-C, Cheng S-E, Chang Y-H, Liu H-J, Hsiang C-Y (2006) Design and biological activities of novel inhibitory peptides for SARS-CoV spike protein and angiotensinconverting enzyme 2 interaction. Antiviral Res 69:70-76. https:// doi.org/10.1016/j.antiviral.2005.10.005

24. Lan J, Ge J, Yu J, Shan S, Zhou H, Fan S, Zhang Q, Shi X, Wang Q, Zhang L, Wang X (2020) Structure of the SARS-CoV-2 spike receptor-binding domain bound to the ACE2 receptor. Nature 581:215-220. https://doi.org/10.1038/s41586-020-2180-5

25. Yan R, Zhang Y, Li Y, Xia L, Guo Y, Zhou Q (2020) Structural basis for the recognition of SARS-CoV-2 by full-length human ACE2. Science 367:1444-1448. https://doi.org/10.1126/scien ce.abb2762

26. Ma TKW, Kam KKH, Yan BP, Lam Y-Y (2010) Renin-angiotensin-aldosterone system blockade for cardiovascular diseases: current status. Br J Pharmacol 160:1273-1292. https://doi.org/10 $.1111 / \mathrm{j} .1476-5381.2010 .00750 . x$

27. Rüster C, Wolf G (2006) Renin-angiotensin-aldosterone system and progression of renal disease. J Am Soc Nephrol 17:29852991. https://doi.org/10.1681/ASN.2006040356

28. Alexander SPH, Armstrong J, Davenport AP, Davies J, Faccenda E, Harding SD, Levi-Schaffer F, Maguire JJ, Pawson AJ, Southan C, Spedding MJ (2020) A rational roadmap for SARS-CoV-2/ COVID-19 pharmacotherapeutic research and development: IUPHAR Review 29. Br J Pharmacol. https://doi.org/10.1111/ bph. 15094

29. Pushpakom S, Iorio F, Eyers PA, Escott KJ, Hopper S, Wells A, Doig A, Guilliams T, Latimer J, McNamee C, Norris A, Sanseau P, Cavalla D, Pirmohamed M (2019) Drug repurposing: progress, challenges and recommendations. Nat Rev Drug Discov 18:41-58. https://doi.org/10.1038/nrd.2018.168

30. Wu C, Liu Y, Yang Y, Zhang P, Zhong W, Wang Y, Wang Q, Xu Y, Li M, Li X, Zheng M, Chen L, Li H (2020) Analysis of therapeutic targets for SARS-CoV-2 and discovery of potential drugs by computational methods. Acta Pharmaceutica Sinica B 10:766-788. https://doi.org/10.1016/j.apsb.2020.02.008

31. Mahdian S, Ebrahim-Habibi A, Zarrabi M (2020) Drug repurposing using computational methods to identify therapeutic options for COVID-19. J Diabetes Metab Disord. https://doi.org/10.1007/ s40200-020-00546-9
32. Sharanya CS, Gangadharan AK, Jayanandhan A, Abdulhameed S, Madathilkovilakathu H (2020) Drug repurposing for COVID19 from FDA approved and experiment stage drugs by in silico methods with SARS CoV-2 spike protein. J Biomol Struct Dyn. https://doi.org/10.26434/chemrxiv.12324980.v1

33. Durdagi S, Aksoydan B, Dogan B, Sahin K, Shahraki A, Birgülİyison N (2020) Screening of clinically approved and investigation drugs as potential inhibitors of SARS-CoV-2 main protease and spike receptor-binding domain bound with ACE2 COVID19 target proteins: a virtual drug repurposing study. J Biomol Struct Dyn. https://doi.org/10.26434/chemrxiv.12032712.v2

34. Trezza A, Iovinelli D, Prischi F, Santucci A, Spiga O (2020) An integrated drug repurposing strategy for the rapid identification of potential SARS-CoV-2 viral inhibitors. Sci Rep. https://doi. org/10.21203/rs.3.rs-24586/v1

35. Chandel V, Sharma PP, Raj S, Rathi B, Kumar D (2020) Structure based drug repurposing through targeting Nsp9 replicase and spike proteins of SARS-CoV-2. J Biomol Struct Dyn. https://doi. org/10.26434/chemrxiv.12292514.v1

36. Choudhary S, Malik YS, Tomar S (2020) Identification of SARS-CoV-2 cell entry inhibitors by drug repurposing using in silico structure-based virtual screening approach. Front Immunol 11:1664. https://doi.org/10.3389/fimmu.2020.01664

37. Cuzzolin A, Sturlese M, Deganutti G, Salmaso V, Sabbadin D, Ciancetta A, Moro S (2016) Deciphering the complexity of ligand-protein recognition pathways using supervised molecular dynamics (SuMD) simulations. J Chem Inf Model 56:687-705. https://doi.org/10.1021/acs.jcim.5b00702

38. Deganutti G, Moro S, Reynolds CA (2020) A supervised molecular dynamics approach to unbiased ligand-protein unbinding. $\mathrm{J}$ Chem Inf Model. https://doi.org/10.1021/acs.jcim.9b01094

39. Sabbadin D, Moro S (2014) Supervised molecular dynamics (SuMD) as a helpful tool to depict GPCR-ligand recognition pathway in a nanosecond time scale. J Chem Inf Model 54:372-376. https://doi.org/10.1021/ci400766b

40. Dolinsky TJ, Nielsen JE, McCammon JA, Baker NA (2004) PDB2PQR: an automated pipeline for the setup of Poisson-Boltzmann electrostatics calculations. Nucleic Acids Res 32:W665-W667. https://doi.org/10.1093/nar/gkh381

41. Olsson MHM, Søndergaard CR, Rostkowski M, Jensen JH (2011) PROPKA3: consistent treatment of internal and surface residues in empirical pK predictions. J Chem Theory Comput 7:525-537. https://doi.org/10.1021/ct100578z

42. Maier JA, Martinez C, Kasavajhala K, Wickstrom L, Hauser KE, Simmerling C (2015) ff14SB: improving the accuracy of protein side chain and backbone parameters from ff $99 \mathrm{SB}$. J Chem Theory Comput 11:3696-3713. https://doi.org/10.1021/acs.jctc.5b00255

43. Jorgensen WL, Chandrasekhar J, Madura JD, Impey RW, Klein ML (1983) Comparison of simple potential functions for simulating liquid water. J Chem Phys 79:926. https://doi. org/10.1063/1.445869

44. Harvey MJ, Giupponi G, Fabritiis GD (2009) ACEMD: accelerating biomolecular dynamics in the microsecond time scale. J Chem Theory Comput 5:1632-1639. https://doi.org/10.1021/ct9000685

45. Forester TR, Smith W (1998) SHAKE, rattle, and roll: efficient constraint algorithms for linked rigid bodies. J. Comput, Chem

46. Kräutler V, van Gunsteren WF, Hünenberger PH (2001) A fast SHAKE algorithm to solve distance constraint equations for small molecules in molecular dynamics simulations. J Comput Chem 22:501-508. https://doi.org/10.1002/1096-987X(20010 415)22:5\%3c501::AID-JCC1021\%3e3.0.CO;2-V

47. Essmann U, Perera L, Berkowitz ML, Darden T, Lee H, Pedersen LG (1995) A smooth particle mesh Ewald method. J Chem Phys 103:8577. https://doi.org/10.1063/1.470117

48. Brenke R, Kozakov D, Chuang G-Y, Beglov D, Hall D, Landon MR, Mattos C, Vajda S (2009) Fragment-based identification 
of druggable "hot spots" of proteins using Fourier domain correlation techniques. Bioinformatics 25:621-627. https://doi. org/10.1093/bioinformatics/btp036

49. Kozakov D, Grove LE, Hall DR, Bohnuud T, Mottarella SE, Luo L, Xia B, Beglov D, Vajda S (2015) The FTMap family of web servers for determining and characterizing ligand-binding hot spots of proteins. Nat Protoc 10:733-755. https://doi.org/10.1038/ nprot.2015.043

50. Jiménez J, Doerr S, Martínez-Rosell G, Rose AS, De Fabritiis G (2017) DeepSite: protein-binding site predictor using 3D-convolutional neural networks. Bioinformatics 33:3036-3042. https:// doi.org/10.1093/bioinformatics/btx350

51. Contini A (2020) Virtual screening of an FDA approved drugs database on two COVID-19 Coronavirus proteins. Life Sci. https ://doi.org/10.26434/chemrxiv.11847381.v1

52. Pettersen EF, Goddard TD, Huang CC, Couch GS, Greenblatt DM, Meng EC, Ferrin TE (2004) UCSF chimera-a visualization system for exploratory research and analysis. J Comput Chem 25:1605-1612. https://doi.org/10.1002/jcc.20084

53. O'Boyle NM, Banck M, James CA, Morley C, Vandermeersch T, Hutchison GR (2011) Open babel: an open chemical toolbox. J Cheminform 3:33. https://doi.org/10.1186/1758-2946-3-33

54. Halgren TA (1996) Merck molecular force field. I. Basis, form, scope, parameterization, and performance of MMFF94. J Comput Chem 17:490-519. https://doi.org/10.1002/(SICI)1096987X(199604)17:5/6\%3c490::AID-JCC1\%3e3.0.CO;2-P

55. Trott O, Olson AJ (2010) AutoDock vina: improving the speed and accuracy of docking with a new scoring function, efficient optimization, and multithreading. J Comput Chem 31:455-461. https://doi.org/10.1002/jcc.21334

56. Wang J, Wang W, Kollman PA, Case DA (2006) Automatic atom type and bond type perception in molecular mechanical calculations. J Mol Graph Model 25:247-260. https://doi.org/10.1016/j. jmgm.2005.12.005

57. Wang J, Wolf RM, Caldwell JW, Kollman PA, Case DA (2004) Development and testing of a general amber force field. J Comput Chem 25:1157-1174. https://doi.org/10.1002/jcc.20035

58. Humphrey W, Dalke A, Schulten K (1996) VMD: visual molecular dynamics. J Mol Graph 14(33-8):27. https://doi. org/10.1016/0263-7855(96)00018-5

59. Miller BR, McGee TD, Swails JM, Homeyer N, Gohlke H, Roitberg AE (2012) MMPBSA.py: an efficient program for end-state free energy calculations. J Chem Theory Comput 8:3314-3321. https://doi.org/10.1021/ct300418h

60. Atanasio S, Deganutti G, Reynolds CA (2020) Addressing free fatty acid receptor 1 (FFAR1) activation using supervised molecular dynamics. J Comput Aided Mol Des. https://doi.org/10.1007/ s10822-020-00338-6

61. Gordon DE, Jang GM, Bouhaddou M, Xu J, Obernier K, White KM, O’Meara MJ, Rezelj VV, Guo JZ, Swaney DL, Tummino TA, Hüttenhain R, Kaake RM, Richards AL, Tutuncuoglu B, Foussard H, Batra J, Haas K, Modak M, Kim M, Haas P, Polacco BJ, Braberg H, Fabius JM, Eckhardt M, Soucheray M, Bennett MJ, Cakir M, McGregor MJ, Li Q, Meyer B, Roesch F, Vallet T, Mac Kain A, Miorin L, Moreno E, Naing ZZC, Zhou Y, Peng S, Shi Y, Zhang Z, Shen W, Kirby IT, Melnyk JE, Chorba JS, Lou K, Dai SA, Barrio-Hernandez I, Memon D, Hernandez-Armenta C, Lyu J, Mathy CJP, Perica T, Pilla KB, Ganesan SJ, Saltzberg DJ, Rakesh R, Liu X, Rosenthal SB, Calviello L, Venkataramanan S, Liboy-Lugo J, Lin Y, Huang X-P, Liu Y, Wankowicz SA, Bohn M, Safari M, Ugur FS, Koh C, Savar NS, Tran QD, Shengjuler D, Fletcher SJ, O'Neal MC, Cai Y, Chang JCJ, Broadhurst DJ, Klippsten S, Sharp PP, Wenzell NA, Kuzuoglu-Ozturk D, Wang H-Y, Trenker R, Young JM, Cavero DA, Hiatt J, Roth TL, Rathore U, Subramanian A, Noack J, Hubert M, Stroud RM, Frankel AD, Rosenberg OS, Verba
KA, Agard DA, Ott M, Emerman M, Jura N, von Zastrow M, Verdin E, Ashworth A, Schwartz O, d'Enfert C, Mukherjee S, Jacobson M, Malik HS, Fujimori DG, Ideker T, Craik CS, Floor SN, Fraser JS, Gross JD, Sali A, Roth BL, Ruggero D, Taunton J, Kortemme T, Beltrao P, Vignuzzi M, García-Sastre A, Shokat KM, Shoichet BK, Krogan NJ (2020) A SARS-CoV-2 protein interaction map reveals targets for drug repurposing. Nature 583:459-468. https://doi.org/10.1038/s41586-020-2286-9

62. Nichols SE, Baron R, Ivetac A, McCammon JA (2011) Predictive power of molecular dynamics receptor structures in virtual screening. J Chem Inf Model 51:1439-1446. https://doi. org/10.1021/ci200117n

63. Salmaso V, Moro S (2018) Bridging molecular docking to molecular dynamics in exploring ligand-protein recognition process: an overview. Front Pharmacol 9:923. https://doi. org/10.3389/fphar.2018.00923

64. Nomura H, Fugono T, Hitaka T, Minami I, Azuma T, Morimoto S, Masuda T (1974) Semisynthetic beta-lactam antibiotics. 6. 1 Sulfocephalosporins and their antipseudomonal activities. J Med Chem 17:1312-1315. https://doi.org/10.1021/jm00258a01 7

65. Hoffmann M, Kleine-Weber H, Schroeder S, Krüger N, Herrler T, Erichsen S, Schiergens TS, Herrler G, Wu N-H, Nitsche A, Müller MA, Drosten C, Pöhlmann S (2020) SARS-CoV-2 cell entry depends on ACE2 and TMPRSS 2 and is blocked by a clinically proven protease inhibitor. Cell 181:271-280.e8. https ://doi.org/10.1016/j.cell.2020.02.052

66. Hoffmann M, Schroeder S, Kleine-Weber H, Müller MA, Drosten C, Pöhlmann S (2020) Nafamostat mesylate blocks activation of SARS-CoV-2: new treatment option for COVID19. Antimicrob Agents Chemother. https://doi.org/10.1128/ AAC.00754-20

67. Sisk JM, Frieman MB, Machamer CE (2018) Coronavirus S protein-induced fusion is blocked prior to hemifusion by Abl kinase inhibitors. J Gen Virol 99:619-630. https://doi.org/10.1099/ jgv.0.001047

68. Coleman CM, Sisk JM, Mingo RM, Nelson EA, White JM, Frieman MB (2016) Abelson kinase inhibitors are potent inhibitors of severe acute respiratory syndrome coronavirus and middle east respiratory syndrome coronavirus fusion. J Virol 90:8924-8933. https://doi.org/10.1128/JVI.01429-16

69. Dyall J, Coleman CM, Hart BJ, Venkataraman T, Holbrook MR, Kindrachuk J, Johnson RF, Olinger GG, Jahrling PB, Laidlaw M, Johansen LM, Lear-Rooney CM, Glass PJ, Hensley LE, Frieman MB (2014) Repurposing of clinically developed drugs for treatment of Middle East respiratory syndrome coronavirus infection. Antimicrob Agents Chemother 58:4885-4893. https://doi. org/10.1128/AAC.03036-14

70. Claghorn JL, Mathew RJ, Mirabi M (1979) Penfluridol: a long acting oral antipsychotic drug. J Clin Psychiatry 40:107-109

71. Lundin A, Dijkman R, Bergström T, Kann N, Adamiak B, Hannoun C, Kindler E, Jónsdóttir HR, Muth D, Kint J, Forlenza M, Müller MA, Drosten C, Thiel V, Trybala E (2014) Targeting membrane-bound viral RNA synthesis reveals potent inhibition of diverse coronaviruses including the middle East respiratory syndrome virus. PLoS Pathog 10:e1004166. https://doi.org/10.1371/ journal.ppat.1004166

72. Bronson J, Black A, Dhar TGM, Ellsworth BA, Merritt JR (2013) To market, to market-2012. Elsevier, Amsterdam, pp 471-546

73. Sabbadin D, Ciancetta A, Moro S (2014) Bridging molecular docking to membrane molecular dynamics to investigate GPCRligand recognition: the human $\mathrm{A}_{2} \mathrm{~A}$ adenosine receptor as a key study. J Chem Inf Model 54:169-183. https://doi.org/10.1021/ ci400532b

74. Wright DB (1986) Cefsulodin. Drug Intell Clin Pharm 20:845849. https://doi.org/10.1177/106002808602001104 
75. Brooke GN, Prischi F (2020) Structural and functional modelling of SARS-CoV-2 entry in animal models. Sci Rep. https://doi. org/10.21203/rs.3.rs-29443/v1

76. Spinello A, Saltalamacchia A, Magistrato A (2020) Is the rigidity of SARS-CoV-2 spike receptor-binding motif the hallmark for its enhanced infectivity? insights from all-atom simulations. J Phys Chem Lett 11:4785-4790. https://doi.org/10.1021/acs.jpcle tt.0c01148
Publisher's Note Springer Nature remains neutral with regard to jurisdictional claims in published maps and institutional affiliations. 